EMBRYARIDDLE
Aeronautical University

SCHOLARLY COMMONS

\section{International Journal of Aviation,} Aeronautics, and Aerospace

\title{
A Mathematical Analysis of the Wind Triangle Problem and an Inquiry of True Airspeed Calculations in Supersonic Flight
}

\author{
Leonard T. Huang \\ University of Nevada, Reno, LeonardHuang@unr.edu \\ Lisa I. Cummings \\ University of Nevada, Reno, Icummings@nevada.unr.edu
}

Follow this and additional works at: https://commons.erau.edu/ijaaa

Part of the Analysis Commons, Navigation, Guidance, Control and Dynamics Commons, and the Numerical Analysis and Computation Commons

\section{Scholarly Commons Citation}

Huang, L. T., \& Cummings, L. I. (2021). A Mathematical Analysis of the Wind Triangle Problem and an Inquiry of True Airspeed Calculations in Supersonic Flight. International Journal of Aviation, Aeronautics, and Aerospace, 8(4). https://doi.org/10.15394/ijaaa.2021.1642

This Article is brought to you for free and open access by the Journals at Scholarly Commons. It has been accepted for inclusion in International Journal of Aviation, Aeronautics, and Aerospace by an authorized administrator of Scholarly Commons. For more information, please contact commons@erau.edu. 


\section{Introduction}

Pilots are familiar with the Wind Triangle Equation, which, for an aircraft flying straight and level, is the vector equation $\vec{G}=\vec{A}+\vec{W}$, where $\vec{G}, \vec{A}$, and $\vec{W}$ are vectors in $\mathbb{R}^{2}$ that are defined as follows:

- $\vec{G}$ is the ground vector of the aircraft, representing the motion of the aircraft with respect to the ground. Its magnitude is called the "ground speed" of the aircraft and denoted by GS, while its compass direction is called the "ground track" of the aircraft and denoted by $\theta$.

- $\vec{A}$ is the air vector of the aircraft, representing the motion of the aircraft with respect to its surrounding air mass. Its magnitude is called the "true air speed" of the aircraft and denoted by TAS, while its compass direction is called the "true heading" of the aircraft and denoted by $\psi$.

- $\vec{W}$ is the wind vector, representing the motion of the aircraft's surrounding air mass with respect to the ground. Its magnitude is called the "wind speed" and denoted by WS, while the compass direction of its negative, $-\vec{W}$, is called the "wind direction" and denoted by $\varphi$.

As such, the Wind Triangle Equation can also be written as the vector equation $\mathrm{GS} \cdot(\sin (\theta), \cos (\theta))=\mathrm{TAS} \cdot(\sin (\psi), \cos (\psi))-\mathrm{WS} \cdot(\sin (\varphi), \cos (\varphi))$.

The most fundamental problem of aerial navigation is the Wind Triangle Problem (WTP), which assumes one of the following three forms:

WTP1. Given $\vec{A}$ and $\vec{W}$, find $\vec{G}$.

WTP2. Given $\vec{G}$ and $\vec{A}$, find $\vec{W}$.

WTP3. Given $\vec{W}$, TAS, and $\theta$, find GS and $\psi$.

Pilots must be well versed in solving WTP3 because it is crucial to flight planning, fuel calculation, and time-of-flight estimation. In flight training, student pilots are taught how to use an E6B flight computer - an analog device having the form of a circular slide rule — to solve WTP3 (Air Training Command, 1973).

It is known that if the value of WS is too large, then WTP3 may not have a solution. Although there is an abundance of literature devoted to an investigation of WTP3, it appears that a mathematically rigorous analysis of the problem is not readily available. For instance, in (Daidzic, 2015, pp. 57-93), the author derives a formula for solving WTP3 but does not discuss when a solution exists. Similarly, both the US Navy's Pilot Trainee Guide and the FAA Handbook's section on the WTP explain the use of the E6B to solve WTP3 but do not address the problem of the existence of solutions (FAA, 2016; US Navy, 2017). Our paper investigated this shortcoming by completely solving the existence problem for WTP3 through carefully stated mathematical results.

In order that our paper does not turn out to be a rehash of well-established results, we shall derive entirely novel mathematical conditions, called "go/no-go conditions", that determine when a solution of WTP3 exists. These conditions can 
be easily tested by a pilot armed with nothing more than a pocket calculator, and we shall explain how these conditions can even be tested graphically.

The calculation of TAS is a vital aspect of evaluating aircraft performance and limitations, especially in high-speed flight. High-performance aircraft usually carry an air data computer (ADC) that calculates and displays TAS, Mach number $(M)$, and calibrated airspeed (CAS) from three critical inputs (which are measured by external sensors): impact air pressure $\left(Q_{\mathrm{c}}\right)$, static air pressure $\left(Q_{\mathrm{s}}\right)$, and total air temperature $\left(T_{\mathrm{t}}\right)$ (Wiolland, 2005, pp. 46-49). Therefore, mathematically speaking, TAS, $M$, and CAS are functions of $Q_{\mathrm{c}}, Q_{\mathrm{s}}$, and $T_{\mathrm{t}}$ in high-speed flight.

However, not every aircraft used in civil aviation possesses an ADC, nor does it have the practical means of directly measuring quantities such as $Q_{\mathrm{c}}$. The following question thus arises: How can a pilot calculate TAS using instrument readings from only a basic flight instrument panel, even at high speeds?

Much has been written about the following mathematical problems:

- Calculating TAS from CAS and pressure altitude $h$ at low speeds (where air compressibility effects can be ignored).

- Calculating TAS from $Q_{\mathrm{c}}, Q_{\mathrm{s}}$, and $T_{\mathrm{t}}$ at high speeds (where air compressibility effects are important).

However, everyday aerospace-engineering discourse seemingly lacks any mention of a function, valid in all flight regimes, that calculates TAS from CAS, $h$, and $T_{\mathrm{t}}$. Brown (2012) delves deeply into the various relationships between these variables and subsequently gives a formula for TAS in supersonic flight, but it still relies on $Q_{\mathrm{c}}$ and $Q_{\mathrm{s}}$ (pp. 30-37).

In the second part of our paper, we shall construct such a function with the goal of providing a method of calculating TAS without directly using $Q_{\mathrm{c}}$ and $Q_{\mathrm{s}}$. Furthermore, we shall provide a rigorous mathematical proof, complete with error estimates, that this method can be combined with fixed-point iteration to compute TAS numerically. Such a rigorous approach seems to be absent from the literature, and our presentation of it is a valuable contribution to the field of aeronautics.

\section{Notation and Terminology}

Throughout this paper, the following notation for intervals shall be used:

- For $a, b \in \mathbb{R}$, let $[a, b[:=\{x \in \mathbb{R} \mid a \leq x<b\}$.

- For $a, b \in \mathbb{R}$, let $] a, b[:=\{x \in \mathbb{R} \mid a<x<b\}$.

For all $a, b \in \mathbb{R}$ satisfying $a<b$, if $I:=\left[a, b\left[\right.\right.$, then define $\operatorname{Mod}_{I}: \mathbb{R} \rightarrow I$ by

$$
\operatorname{Mod}_{I}(x):=\text { The unique } y \in I \text { such that } \frac{x-y}{b-a} \in \mathbb{Z} \text {, }
$$

which translates every real number $x$ to a real number $y$ in $I$, necessarily unique, by integer multiples of the length of $I$.

The Mathematical Wind Triangle Problem

The following definition provides a mathematical formulation of the third form of the WTP (previously referred to as "WTP3"). 
Definition 1. A mathematical WTP3 is defined as an element (TAS,WS, $\theta, \varphi$ ) of the set

$$
\mathbb{R}_{>0} \times \mathbb{R}_{\geq 0} \times\left[0^{\circ}, 360^{\circ}\left[\times\left[0^{\circ}, 360^{\circ}[\right.\right.\right.
$$

in which case a solution of (TAS,WS, $\theta, \varphi)$ is defined as an element (GS, $\psi$ ) of $\mathbb{R}_{>0} \times\left[0^{\circ}, 360^{\circ}[\right.$ that satisfies the following vector equation:

$\mathrm{GS} \cdot(\sin (\theta), \cos (\theta))=\mathrm{TAS} \cdot(\sin (\psi), \cos (\psi))-\mathrm{WS} \cdot(\sin (\varphi), \cos (\varphi))$.

Remark: The reason for demanding that TAS be a positive real number is that an aircraft must be in motion with respect to its surrounding air mass for flight to be possible. Likewise, the reason for demanding that GS be a positive real number is that an aircraft must be able to travel to its planned destination.

Let us state two basic mathematical theorems that form the foundation of our derivation of the go/no-go conditions.

Theorem 1. Let $b, c \in \mathbb{R}$. Then the following three statements are true:

1. There are at most two $x \in \mathbb{R}_{>0}$ such that $x^{2}+b x+c=0$.

2. There is at least one $x \in \mathbb{R}_{>0}$ such that $x^{2}+b x+c=0$ if and only if the inequalities $0 \leq b^{2}-4 c$ and $b<\sqrt{b^{2}-4 c}$ hold, in which case the largest such $x$ is equal to $\frac{-b+\sqrt{b^{2}-4 c}}{2}$.

3. There are exactly two $x \in \mathbb{R}_{>0}$ such that $x^{2}+b x+c=0$ if and only if the inequalities $0<b^{2}-4 c$ and $b<-\sqrt{b^{2}-4 c}$ hold, in which case the smallest such $x$ is equal to $\frac{-b-\sqrt{b^{2}-4 c}}{2}$ and the largest such $x$ is equal to $\frac{-b+\sqrt{b^{2}-4 c}}{2}$.

Proof. Theorem 1 is a straightforward consequence of the quadratic formula, so we shall omit a proof.

Theorem 2. Let $\vec{v} \in \mathbb{R}^{2}$ and $a \in \mathbb{R}_{>0}$. Then there is an $\alpha \in\left[0^{\circ}, 360^{\circ}\right.$, which is necessarily unique, such that $\vec{v}=(a \sin (\alpha), a \cos (\alpha))$ if and only if $\|\vec{v}\|^{2}=a^{2}$. Proof. Theorem 2 is a straightforward consequence of the Pythagorean Identity, so we shall omit a proof as well.

In order to ensure a smooth transition from Theorems 1 and 2 to our main results, two preparatory lemmas are required.

Lemma 1. Let (TAS,WS, $\theta, \varphi$ ) be a mathematical WTP3. Then for every GS $\in$ $\mathbb{R}_{>0}$, the following two statements are equivalent:

1. There is a $\psi \in\left[0^{\circ}, 360^{\circ}\right.$ [ such that (GS, $\psi$ ) is a solution of (TAS,WS, $\theta, \varphi$ ).

2. $\mathrm{GS}^{2}+2 \mathrm{WS} \cos (\varphi-\theta) \cdot \mathrm{GS}+\left(\mathrm{WS}^{2}-\mathrm{TAS}^{2}\right)=0$.

Proof. Let GS $\in \mathbb{R}_{>0}$. Then, by Definition 1 , there is a $\psi \in\left[0^{\circ}, 360^{\circ}[\right.$ such that (GS, $\psi$ ) is a solution of (TAS,WS, $\theta, \varphi)$ if and only if

$\mathrm{TAS} \cdot(\sin (\psi), \cos (\psi))=\mathrm{GS} \cdot(\sin (\theta), \cos (\theta))+\mathrm{WS} \cdot(\sin (\varphi), \cos (\varphi))$, which, by Theorem 2 , is true if and only if

$$
\mathrm{TAS}^{2}=\|\mathrm{GS} \cdot(\sin (\theta), \cos (\theta))+\mathrm{WS} \cdot(\sin (\varphi), \cos (\varphi))\|^{2},
$$

which is equivalent to

$$
\mathrm{GS}^{2}+2 \mathrm{WS} \cos (\varphi-\theta) \cdot \mathrm{GS}+\left(\mathrm{WS}^{2}-\mathrm{TAS}^{2}\right)=0
$$


The proof of Lemma 1 is complete.

Lemma 2. Let (TAS,WS, $\theta, \varphi$ ) be a mathematical WTP3. Then the following three statements are true:

1. (TAS,WS, $\theta, \varphi$ ) has at most two solutions; if it has two solutions, then their first coordinates are distinct.

2. (TAS,WS, $\theta, \varphi$ ) has a solution if and only if the following conditions hold:

(a) $0 \leq \mathrm{TAS}^{2}-\mathrm{WS}^{2} \sin ^{2}(\theta-\varphi)$.

(b) $\mathrm{WS} \cos (\varphi-\theta)<\sqrt{\mathrm{TAS}^{2}-\mathrm{WS}^{2} \sin ^{2}(\varphi-\theta)}$.

3. (TAS,WS, $\theta, \varphi$ ) has two solutions if and only if the following conditions hold:

(a) $0<\mathrm{TAS}^{2}-\mathrm{WS}^{2} \sin ^{2}(\theta-\varphi)$.

(b) $\mathrm{WS} \cos (\varphi-\theta)<-\sqrt{\mathrm{TAS}^{2}-\mathrm{WS}^{2} \sin ^{2}(\varphi-\theta)}$.

Proof. If (GS, $\psi$ ) is a solution of (TAS,WS, $\theta, \varphi$ ), then, by Statement 1 of Lemma 1 and Theorem 1, there are at most two possible values of GS.

In addition, if (GS, $\psi)$ and $\left(\mathrm{GS}, \psi^{\prime}\right)$ are solutions of (TAS,WS, $\left.\theta, \varphi\right)$, then $\mathrm{TAS} \cdot(\sin (\psi), \cos (\psi))=\mathrm{GS} \cdot(\sin (\theta), \cos (\theta))+\mathrm{WS} \cdot(\sin (\varphi), \cos (\varphi))$,

$\mathrm{TAS} \cdot\left(\sin \left(\psi^{\prime}\right), \cos \left(\psi^{\prime}\right)\right)=\mathrm{GS} \cdot(\sin (\theta), \cos (\theta))+\mathrm{WS} \cdot(\sin (\varphi), \cos (\varphi))$, which, by the uniqueness clause of Theorem 2, means that $\psi=\psi^{\prime}$. Consequently, (TAS,WS, $\theta, \varphi$ ) has at most two solutions, and if it has two solutions, then their first coordinates are distinct. Hence, Statement 1 is true.

Suppose that (TAS,WS, $\theta, \varphi$ ) has a solution. By Lemma 1 , there is a GS $\in$ $\mathbb{R}_{>0}$ such that

$$
\mathrm{GS}^{2}+2 \mathrm{WS} \cos (\varphi-\theta) \cdot \mathrm{GS}+\left(\mathrm{WS}^{2}-\mathrm{TAS}^{2}\right)=0
$$

Statement 2 of Theorem 1 then implies Conditions 2(a) and 2(b).

Conversely, suppose that Conditions 2(a) and 2(b) hold. By Statement 2 of Theorem 1 , there is a GS $\in \mathbb{R}_{>0}$ such that

$$
\mathrm{GS}^{2}+2 \mathrm{WS} \cos (\varphi-\theta) \cdot \mathrm{GS}+\left(\mathrm{WS}^{2}-\mathrm{TAS}^{2}\right)=0
$$

Lemma 1 then implies that (TAS,WS, $\theta, \varphi$ ) has a solution.

Hence, Statement 2 is true.

Next, suppose that (TAS,WS, $\theta, \varphi$ ) has two solutions. By Lemma 1 and Statement 1 of this lemma, there are two GS $\in \mathbb{R}_{>0}$ such that

$$
\mathrm{GS}^{2}+2 \mathrm{WS} \cos (\varphi-\theta) \cdot \mathrm{GS}+\left(\mathrm{WS}^{2}-\mathrm{TAS}^{2}\right)=0
$$

Statement 3 of Theorem 1 then implies Conditions 3(a) and 3(b).

Conversely, suppose that Conditions 3(a) and 3(b) hold. By Statement 3 of

Theorem 1, there are two GS $\in \mathbb{R}_{>0}$ such that

$$
\mathrm{GS}^{2}+2 \mathrm{WS} \cos (\varphi-\theta) \cdot \mathrm{GS}+\left(\mathrm{WS}^{2}-\mathrm{TAS}^{2}\right)=0
$$

Lemma 1 then implies that (TAS,WS, $\theta, \varphi$ ) has two solutions.

Hence, Statement 3 is true, which completes the proof of Lemma 2. 
The go/no-go conditions are basically a pair of geometrical conditions that are defined in terms of a certain subset of $\mathbb{R}^{2}$, which we shall now specify.

Definition 2. Define a subset $S$ of $\mathbb{R}^{2}$ by

$$
(x, y) \in S \Leftrightarrow\left\{\begin{array}{c}
x=-1 \text { and } 0 \leq y ; \text { or } \\
-1<x<0 \text { and } 0 \leq y \leq \frac{1}{\sqrt{1-x^{2}}} ; \text { or } \\
0 \leq x \leq 1 \text { and } 0 \leq y<1 .
\end{array}\right.
$$

This subset can be visualized in Figure 1 .

\section{Figure 1}

\section{A Graphical Representation of $S$}

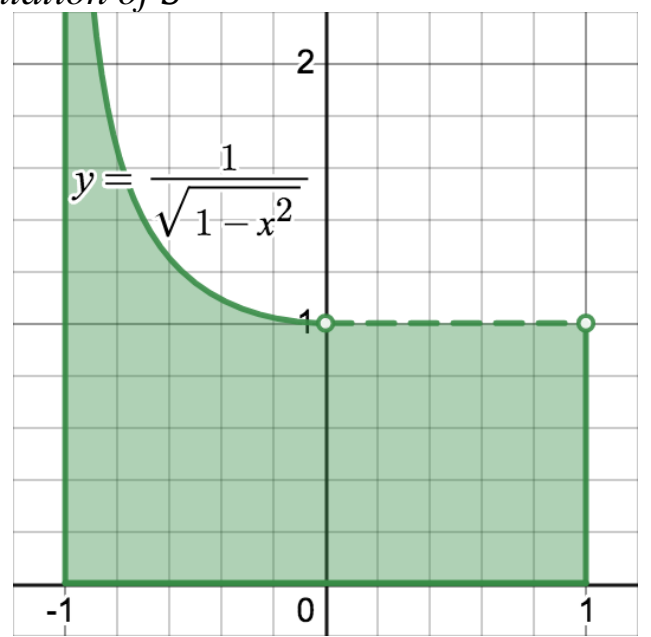

Note. The region $S$ is represented by the green area.

Proposition 1. Let (TAS,WS, $\theta, \varphi$ ) be a mathematical WTP3. Then the following two statements are true:

1. (TAS,WS, $\theta, \varphi)$ has a solution if and only if $\left(\cos (\varphi-\theta), \frac{\mathrm{WS}}{\mathrm{TAS}}\right) \in S$.

The condition $\left(\cos (\varphi-\theta), \frac{\mathrm{WS}}{\mathrm{TAS}}\right) \in S$ is called the "go condition".

2. (TAS,WS, $\theta, \varphi)$ has no solution if and only if $\left(\cos (\varphi-\theta), \frac{\mathrm{WS}}{\mathrm{TAS}}\right) \notin S$.

The condition $\left(\cos (\varphi-\theta), \frac{\mathrm{WS}}{\mathrm{TAS}}\right) \notin S$ is called the "no-go condition".

Proof. It suffices to prove Statement 1 as Statement 2 is logically equivalent to it.

By Lemma 2, (TAS,WS, $\theta, \varphi$ ) has a solution if and only if its Conditions 2(a) and 2(b) hold, which are equivalent to the following two conditions, respectively:

1. $\left(\frac{\mathrm{WS}}{\mathrm{TAS}}\right)^{2} \sin ^{2}(\varphi-\theta) \leq 1$ or, equivalently, $\frac{\mathrm{WS}}{\mathrm{TAS}} \sqrt{1-\cos ^{2}(\varphi-\theta)} \leq 1$.

2'. $\frac{\mathrm{WS}}{\mathrm{TAS}} \cos (\varphi-\theta)<\sqrt{1-\left(\frac{\mathrm{WS}}{\mathrm{TAS}}\right)^{2} \sin ^{2}(\varphi-\theta)}$. 
When $\frac{\mathrm{WS}}{\mathrm{TAS}}=0$, Conditions 1' and 2' automatically hold, so suppose henceforth that $0<\frac{\mathrm{WS}}{\mathrm{TAS}}$. Three cases are now to be considered:

- $\cos (\varphi-\theta)=-1$, in which case Conditions 1' and 2' automatically hold.

- $-1<\cos (\varphi-\theta)<0$, in which case Condition 2' automatically holds and Condition 1' holds if and only if $\frac{\mathrm{WS}}{\mathrm{TAS}} \leq \frac{1}{\sqrt{1-\cos ^{2}(\varphi-\theta)}}$.

- $0 \leq \cos (\varphi-\theta) \leq 1$, in which case Condition 2 ' holds if and only if $\frac{\mathrm{WS}}{\mathrm{TAS}}<1$, which guarantees that Condition 1' also holds.

Therefore, (TAS,WS, $\theta, \varphi)$ has a solution if and only if $\left(\cos (\varphi-\theta), \frac{\mathrm{WS}}{\mathrm{TAS}}\right) \in S$. Remark: The cases $\cos (\varphi-\theta)=-1, \cos (\varphi-\theta)=0$, and $\cos (\varphi-\theta)=1$ represent a pure tailwind, a pure crosswind, and a pure headwind, respectively. Proposition 2. Let (TAS,WS, $\theta, \varphi$ ) be a solvable mathematical WTP3. Then the following statements are true:

1. $0 \leq \mathrm{TAS}^{2}-\mathrm{WS}^{2} \sin ^{2}(\theta-\varphi)$ or, equivalently, $\frac{\mathrm{WS}}{\mathrm{TAS}} \sin (\varphi-\theta) \in[-1,1]$.

2. If (TAS,WS, $\theta, \varphi$ ) has two solutions and (GS, $\psi$ ) denotes the solution with the smallest first coordinate, then the following conditions hold:
(a) $\mathrm{GS}=-\mathrm{WS} \cos (\varphi-\theta)-\sqrt{\mathrm{TAS}^{2}-\mathrm{WS}^{2} \sin ^{2}(\varphi-\theta)}$.
(b) $\Delta:=\operatorname{Mod}_{\left[-180^{\circ}, 180^{\circ}[\right.}(\psi-\theta) \in\left[-180^{\circ},-90^{\circ}[\cup] 90^{\circ}, 180^{\circ}[\right.$.

3. In all cases, if (GS, $\psi$ ) denotes the solution of (TAS, WS, $\theta, \varphi$ ) with the largest first coordinate, then the following conditions hold:
(a) $\mathrm{GS}=-\mathrm{WS} \cos (\varphi-\theta)+\sqrt{\mathrm{TAS}^{2}-\mathrm{WS}^{2} \sin ^{2}(\varphi-\theta)}$.
(b) $\Delta:=\operatorname{Mod}_{\left[-180^{\circ}, 180^{\circ}\right.}(\psi-\theta) \in\left[-90^{\circ}, 90^{\circ}\right]$.
(c) $\psi=\operatorname{Mod}_{\left[0^{\circ}, 360^{\circ}[\right.}\left(\theta+\sin ^{-1}\left(\frac{\mathrm{WS}}{\mathrm{TAS}} \sin (\varphi-\theta)\right)\right)$.

Note: $\Delta$ is often called the "wind-correction angle (WCA)".

Proof. Statement 1 is an immediate consequence of Condition 2(a) of Lemma 2.

Suppose that (TAS,WS, $\theta, \varphi$ ) has two solutions and that (GS, $\psi$ ) denotes the solution with the smallest first coordinate. Then by Lemma 1, Statement 1 of Lemma 2, and Statement 3 of Theorem 1,

$$
\mathrm{GS}=-\mathrm{WS} \cos (\varphi-\theta)-\sqrt{\mathrm{TAS}^{2}-\mathrm{WS}^{2} \sin ^{2}(\varphi-\theta)},
$$

which proves Statement 2(a). To prove Statements 2(b), first use the identities

$$
\begin{aligned}
\text { TAS } \sin (\psi) & =\mathrm{GS} \sin (\theta)+\mathrm{WS} \sin (\varphi), \\
\mathrm{TAS} \cos (\psi) & =\mathrm{GS} \cos (\theta)+\mathrm{WS} \cos (\varphi),
\end{aligned}
$$

to obtain the following four identities:

1. TAS $\sin (\psi) \sin (\theta)=\mathrm{GS} \sin ^{2}(\theta)+\mathrm{WS} \sin (\varphi) \sin (\theta)$. 
2. TAS $\cos (\psi) \cos (\theta)=\mathrm{GS} \cos ^{2}(\theta)+\mathrm{WS} \cos (\varphi) \cos (\theta)$.

3. TAS $\cos (\theta) \sin (\psi)=\mathrm{GS} \cos (\theta) \sin (\theta)+\mathrm{WS} \cos (\theta) \sin (\varphi)$.

4. TAS $\cos (\psi) \sin (\theta)=\mathrm{GS} \cos (\theta) \sin (\theta)+\mathrm{WS} \cos (\varphi) \sin (\theta)$.

Adding Identities 1 and 2, and applying Condition 3(a) of Lemma 2, we get

$$
\mathrm{TAS} \cos (\psi-\theta)=\mathrm{GS}+\mathrm{WS} \cos (\varphi-\theta)=-\sqrt{\mathrm{TAS}^{2}-\mathrm{WS}^{2} \sin ^{2}(\varphi-\theta)}<0 .
$$

Then as TAS is positive, Statement 2(b) is thus obtained.

Finally, suppose that (GS, $\psi$ ) denotes the solution of (TAS,WS, $\theta, \varphi$ ) with the largest first coordinate, regardless of the number of solutions. Then by Lemma 1 and Statement 2 of Theorem 1,

$$
\mathrm{GS}=-\mathrm{WS} \cos (\varphi-\theta)+\sqrt{\mathrm{TAS}^{2}-\mathrm{WS}^{2} \sin ^{2}(\varphi-\theta)},
$$

which proves Statement 3(a). Adding Identities 1 and 2, we get

$$
\mathrm{TAS} \cos (\psi-\theta)=\mathrm{GS}+\mathrm{WS} \cos (\varphi-\theta)=\sqrt{\mathrm{TAS}^{2}-\mathrm{WS}^{2} \sin ^{2}(\varphi-\theta)} \geq 0 .
$$

Then as TAS is positive, Statement $3(\mathrm{~b})$ is obtained. Now, subtracting Identity 4 from Identity 3 yields TAS $\sin (\psi-\theta)=$ WS $\sin (\varphi-\theta)$, which, together with the $360^{\circ}$-periodicity of the sine function, implies that

$$
\text { TAS } \sin \left(\operatorname{Mod}_{\left[-180^{\circ}, 180^{\circ}[\right.}(\psi-\theta)\right)=\mathrm{WS} \sin (\varphi-\theta) .
$$

As $\operatorname{Mod}_{\left[-180^{\circ}, 180^{\circ}\right.}(\psi-\theta) \in\left[-90^{\circ}, 90^{\circ}\right]$, it then follows that

Therefore,

$$
\operatorname{Mod}_{\left[-180^{\circ}, 180^{\circ}[\right.}(\psi-\theta)=\sin ^{-1}\left(\frac{\mathrm{WS}}{\mathrm{TAS}} \sin (\varphi-\theta)\right) \text {. }
$$

$$
\begin{aligned}
\psi & =\operatorname{Mod}_{\left[0^{\circ}, 360^{\circ}[\right.}(\psi) \\
& =\operatorname{Mod}_{\left[0^{\circ}, 360^{\circ}[\right.}(\theta+(\psi-\theta)) \\
& =\operatorname{Mod}_{\left[0^{\circ}, 360^{\circ}[\right.}\left(\theta+\operatorname{Mod}_{\left[-180^{\circ}, 180^{\circ}[\right.}(\psi-\theta)\right) \\
& =\operatorname{Mod}_{\left[0^{\circ}, 360^{\circ}[\right.}\left(\theta+\sin ^{-1}\left(\frac{\mathrm{WS}}{\mathrm{TAS}} \sin (\varphi-\theta)\right)\right),
\end{aligned}
$$

which proves Statement 3(c).

Remark: For a mathematical WTP3 (TAS,WS, $\theta, \varphi$ ) with two solutions, the one with the largest first coordinate (representing the largest groundspeed) is the most desirable because an aircraft's time of flight to its planned destination must be as short as possible. By Proposition 2, the solution (GS, $\psi$ ) of (TAS,WS, $\theta, \varphi$ ) with the smallest first coordinate satisfies the WCA condition

$$
\Delta \in\left[-180^{\circ},-90^{\circ}[\mathrm{U}] 90^{\circ}, 180^{\circ}[\text {, }\right.
$$

which means that the aircraft is pointing away from its planned destination. This is clearly an inefficient - hence undesirable - solution. 
Example 1. A pilot intends to fly a ground track of $70^{\circ}$ at a true airspeed of $95 \mathrm{kt}$. At the desired operating altitude, the windspeed is $20 \mathrm{kt}$ and the wind direction is $340^{\circ}$. The mathematical WTP3 corresponding to this flight plan is

$$
\text { (TAS,WS, } \theta, \varphi)=\left(95 \mathrm{kt}, 20 \mathrm{kt}, 70^{\circ}, 340^{\circ}\right) \text {. }
$$

As $\cos (\varphi-\theta)=0$ and $\frac{\mathrm{WS}}{\mathrm{TAS}}=0.21 \ldots$, this mathematical WTP3 satisfies the go condition, so it has a solution (see Figure 2).

\section{Figure 2}

\section{The Go Condition Satisfied in Example 1}

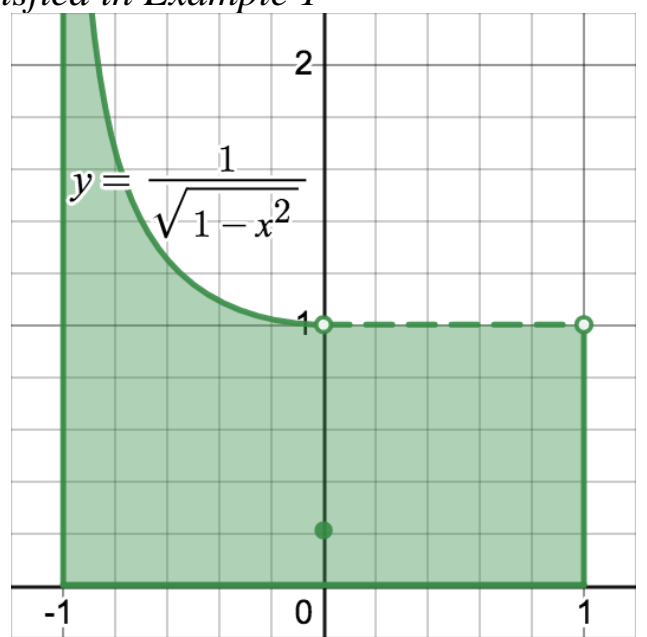

Note. The go condition is represented by the green solid dot.

If we denote by $(\mathrm{GS}, \psi)$ the solution with the largest first coordinate, then we have, by Statement 3 of Proposition 2,

$$
\begin{aligned}
\mathrm{GS} & =-\mathrm{WS} \cos (\varphi-\theta)+\sqrt{\mathrm{TAS}^{2}-\mathrm{WS}^{2} \sin ^{2}(\varphi-\theta)}=92.87 \ldots, \\
\psi & =\operatorname{Mod}_{\left[0^{\circ}, 360^{\circ}[\right.}\left(\theta+\sin ^{-1}\left(\frac{\mathrm{WS}}{\mathrm{TAS}} \sin (\varphi-\theta)\right)\right)=57.84 \ldots \circ, \\
\Delta & =\operatorname{Mod}_{\left[-180^{\circ}, 180^{\circ}[\right.}(\psi-\theta)=-12.15 \ldots \circ .
\end{aligned}
$$

Example 2. A pilot intends to fly a ground track of $80^{\circ}$ at a true airspeed of $80 \mathrm{kt}$. At the desired operating altitude, the windspeed is $120 \mathrm{kt}$ and the wind direction is $120^{\circ}$. The mathematical WTP3 corresponding to this scenario is

$(\mathrm{TAS}, \mathrm{WS}, \theta, \varphi)=\left(80 \mathrm{kt}, 120 \mathrm{kt}, 80^{\circ}, 120^{\circ}\right)$.

As $\cos (\varphi-\theta)=0.76 \ldots$ and $\frac{\mathrm{WS}}{\mathrm{TAS}}=1.5$, this mathematical WTP3 satisfies the no-go condition, so it has no solution (see Figure 3 ). 


\section{Figure 3}

\section{The No-Go Condition Satisfied in Example 2}

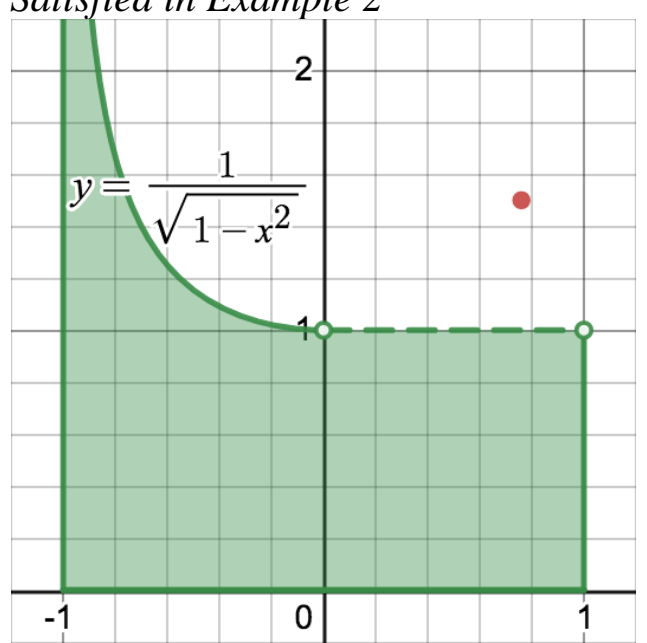

Note. The no-go condition is represented by the red solid dot.

\section{Computing True Airspeed from Basic Instrument Readings}

In this section, we shall apply the fluid mechanics of compressible air to derive a function that calculates TAS from calibrated airspeed, pressure altitude, and total air temperature, in both subsonic and supersonic flight.

Firstly, let us specify the values of some fundamental physical constants:

- The sea-level acceleration of gravity: $g_{0}:=9.80665 \mathrm{~m} / \mathrm{s}$.

- The adiabatic index of air: $\gamma:=\frac{7}{5}$.

- The tropospheric temperature lapse rate: $L_{0}:=0.0065 \mathrm{~K} / \mathrm{m}$.

- The molar mass of air: $M:=0.0289644 \mathrm{~kg} / \mathrm{mol}$.

- The standard sea-level atmospheric pressure: $P_{0}:=101325 \mathrm{~N} / \mathrm{m}^{2}$.

- The gas constant: $R^{*}:=8.31432 \frac{\mathrm{N} \cdot \mathrm{m}}{\mathrm{mol} \cdot \mathrm{K}}$.

- The standard sea-level temperature: $T_{0}:=288.15 \mathrm{~K}$.

- These definitions are obtained from the U.S. Standard Atmosphere 1976.

Next, let us also specify the values of some derived physical constants:

- The standard sea-level speed of sound: $a_{0}:=\sqrt{\frac{\gamma R^{*} T_{0}}{M}}$.

- The standard atmospheric pressure at $11000 \mathrm{~m}: P_{0}:=\left(1+\frac{L_{0} \cdot 11000 \mathrm{~m}}{T_{0}}\right)^{-\frac{g_{0} M}{R^{*} L_{0}}}$.

- The standard temperature at $11000 \mathrm{~m}: T_{1}:=T_{0}-L_{0} \cdot 11000 \mathrm{~m}=216.65 \mathrm{~K}$.

In fluid mechanics, an important result of applying the Bernoulli Equation to compressible air is that subsonic Mach numbers $M$ (i.e., $M \in[0,1]$ ) are in one- 
to-one correspondence with values of the ratio $\frac{Q_{\mathrm{c}}}{P}$ in the interval $\left[0,\left(\frac{6}{5}\right)^{7 / 2}-1\right]$ via the following equation (Porter \& Hartman, 1979, pp. 83-88):

$$
M=\sqrt{\frac{2}{\gamma-1}\left[\left(\frac{Q_{\mathrm{c}}}{P}+1\right)^{\frac{\gamma-1}{\gamma}}-1\right]}=\sqrt{5\left[\left(\frac{Q_{\mathrm{c}}}{P}+1\right)^{\frac{2}{7}}-1\right]} .
$$

The critical value of $\left(\frac{6}{5}\right)^{7 / 2}-1$ for the ratio $\frac{Q_{\mathrm{c}}}{P}$ determines the boundary between subsonic and supersonic air flows.

According to the Rayleigh Supersonic Pitot Equation, supersonic Mach numbers $M$ (i.e., $M \in \mathbb{R}_{\geq 1}$ ) are in one-to-one correspondence with values of the ratio $\frac{Q_{\mathrm{c}}}{P}$ in the interval $\left[\left(\frac{6}{5}\right)^{7 / 2}-1, \infty[\right.$ via the following equation (Miller \& Veltin, 2012, pp. 235-246):

$$
\frac{Q_{\mathrm{c}}}{P}+1=\left(\frac{\gamma+1}{2} M^{2}\right)^{\frac{\gamma}{\gamma-1}}\left(\frac{\gamma+1}{2 \gamma M^{2}-\gamma+1}\right)^{\frac{1}{\gamma-1}}=\frac{6^{5 / 2}\left(\frac{6}{5}\right)^{7 / 2} M^{7}}{\left(7 M^{2}-1\right)^{5 / 2}} .
$$

In order to combine these two equations relating $M$ to $\frac{Q_{\mathrm{c}}}{P}$ into a single one, let us introduce a useful mathematical function.

Definition 3. Define the function $f: \mathbb{R}_{\geq 0} \rightarrow \mathbb{R}_{\geq 0}$ by

$$
f(x):=\left\{\begin{array}{l}
\left(1+\frac{x^{2}}{5}\right)^{\frac{7}{2}}-1, \text { if } 0 \leq x \leq 1 \\
\frac{6^{5 / 2}\left(\frac{6}{5}\right)^{7 / 2} x^{7}}{\left(7 x^{2}-1\right)^{5 / 2}}-1, \text { if } 1 \leq x .
\end{array}\right.
$$

The function $f$ is twice continuously differentiable; in fact,

$$
f^{\prime}(x)=\left\{\begin{array}{c}
\frac{7}{5} x\left(1+\frac{x^{2}}{5}\right)^{\frac{5}{2}}, \text { if } 0 \leq x \leq 1 \\
\frac{6^{5 / 2}\left(\frac{6}{5}\right)^{7 / 2} 7 x^{6}\left(2 x^{2}-1\right)}{\left(7 x^{2}-1\right)^{7 / 2}}, \text { if } 1 \leq x ;
\end{array}\right.
$$




$$
f^{\prime \prime}(x)=\left\{\begin{array}{l}
\frac{7}{5}\left(1+\frac{6 x^{2}}{5}\right)\left(1+\frac{x^{2}}{5}\right)^{\frac{3}{2}}, \text { if } 0 \leq x \leq 1 ; \\
\frac{6^{5 / 2}\left(\frac{6}{5}\right)^{7 / 2} 7 x^{5}\left(14 x^{4}-9 x^{2}+6\right)}{\left(7 x^{2}-1\right)^{9 / 2}}, \text { if } 1 \leq x .
\end{array}\right.
$$

Hence, $f$ has an excellent degree of smoothness. Furthermore, as $f(0)=0$ and $f^{\prime}$ is positive on $\mathbb{R}_{>0}$, we find that $f$ is increasing with range $\mathbb{R}_{\geq 0}$. For a graph of $f$, see Figure 4.

\section{Figure 4}

A Graph of $f$

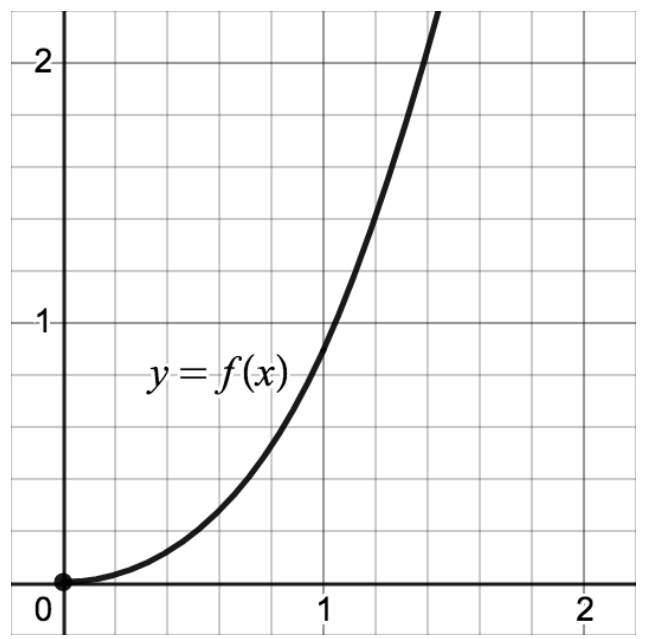

As promised, with the help of $f$, the two equations relating $M$ to $\frac{Q_{\mathrm{c}}}{P}$, for subsonic and supersonic air flows, can be nicely combined into a single equation:

$$
\text { (*) } \quad \frac{Q_{\mathrm{c}}}{P}=f(M) .
$$

Equation (*) is the key to the construction of a function that calculates TAS from only calibrated airspeed, pressure altitude, and total air temperature.

\section{Static Air Pressure as a Function of Pressure Altitude}

Most flights take place at pressure altitudes ranging from $0 \mathrm{~m}$ to $20000 \mathrm{~m}$, so we shall define static air pressure as a function of pressure altitude in the range [0 m, $20000 \mathrm{~m}[$, in accordance with the U.S. Standard Atmosphere 1976.

Definition 4. Static air pressure is the function $P:\left[0 \mathrm{~m}, 20000 \mathrm{~m}[\rightarrow] 0, P_{0}\right]$ defined by 


$$
P(h):=\left\{\begin{array}{c}
P_{0}\left(1+\frac{L_{0} h}{T_{0}}\right)^{-\frac{g_{0} M}{R^{*} L_{0}}}, \text { if } 0 \mathrm{~m} \leq h \leq 11000 \mathrm{~m} ; \\
P_{1} e^{-g_{0} M(h-11000 \mathrm{~m}) / R^{*} T_{1}}, \text { if } 11000 \mathrm{~m} \leq h \leq 20000 \mathrm{~m} .
\end{array}\right.
$$

Note that $P$ is strictly increasing and continuous.

\section{Impact Air Pressure as a Function of Calibrated Airspeed}

An airspeed indicator on a high-performance aircraft establishes a one-toone correspondence between impact air pressures and calibrated airspeeds, under the assumption that the aircraft is flying at sea level (see Figure 5).

\section{Figure 5}

The Instrument Panel of the Rocket-Powered X-15

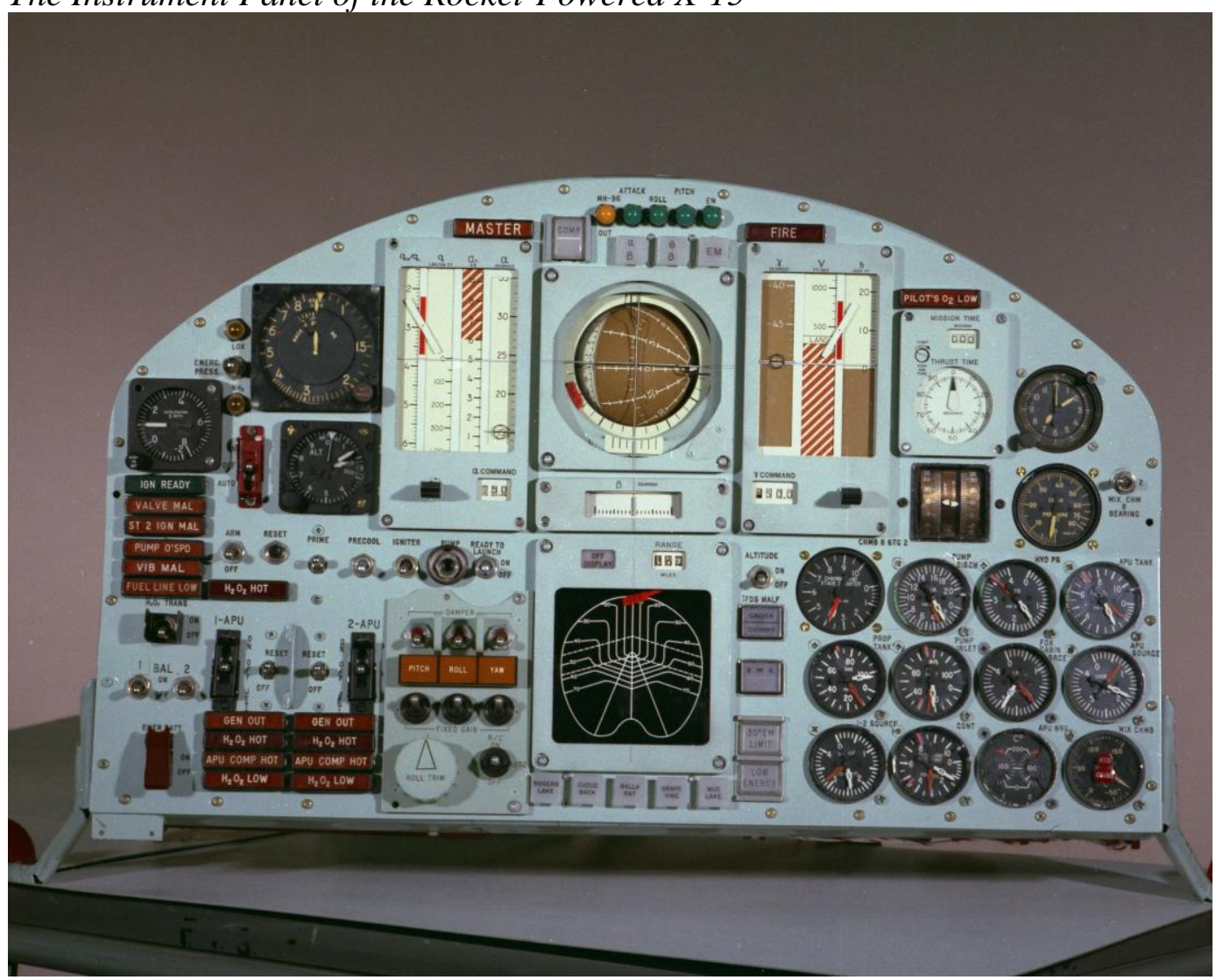

Note. The airspeed indicator and Machmeter are located in the upper left corner of the panel. equation:

It follows from Equation (*) that $Q_{\mathrm{c}}$ and CAS are related via the following

$$
\frac{Q_{\mathrm{c}}}{P_{0}}=f\left(\frac{\mathrm{CAS}}{a_{0}}\right)
$$


This allows us to define impact air pressure as a function of calibrated airspeed.

Definition 5. Impact air pressure is the function $Q_{c}: \mathbb{R}_{\geq 0} \rightarrow \mathbb{R}_{\geq 0}$ defined by

$$
Q_{\mathrm{c}}(\mathrm{CAS}):=P_{0} f\left(\frac{\mathrm{CAS}}{a_{0}}\right) \text {. }
$$

Note that $Q_{\mathrm{c}}$ is strictly increasing, bijective, and twice continuously differentiable.

\section{Mach Number as a Function of Calibrated Airspeed and Pressure Altitude}

Equation (*) and the two functions $P$ and $Q_{\mathrm{c}}$ now allow us to define Mach number as a function of calibrated airspeed and pressure altitude.

Definition 6. Mach number is the function $M: \mathbb{R}_{\geq 0} \times\left[0 \mathrm{~m}, 20000 \mathrm{~m}\left[\rightarrow \mathbb{R}_{\geq 0}\right.\right.$ defined by

$$
M(\mathrm{CAS}, h):=f^{-1}\left(\frac{Q_{\mathrm{c}}(\mathrm{CAS})}{P(h)}\right) .
$$

Note that $M$ is continuous as $f^{-1}$ is continuous.

\section{Inverting the Function $f$}

It is clear from Definition 6 that knowing how to compute $f^{-1}$ is crucial. An explicit formula for $f^{-1}$ on the interval $\left[0,\left(\frac{6}{5}\right)^{7 / 2}-1\right]$ is as follows:

$$
f^{-1}(y)=\sqrt{5\left((y+1)^{2 / 7}-1\right)}
$$

However, an explicit formula for $f^{-1}$ on the interval $\left[\left(\frac{6}{5}\right)^{7 / 2}-1, \infty[\right.$ appears to be hard to find, so we must resort to numerical techniques. For convenience, we shall employ the technique of fixed-point iteration, and it is our current objective to prove the convergence of this technique and provide error estimates.

Letting $J:=\left[\left(\frac{6}{5}\right)^{7 / 2}-1, \infty\left[\right.\right.$, we can define a $J$-indexed family $\left(g_{r}\right)_{r \in J}$ of functions from $\mathbb{R}_{\geq 1}$ to $\mathbb{R}_{\geq 0}$ by

$$
\forall r \in J: \quad g_{r}(x):=\left(\frac{7}{6}\right)^{\frac{5}{4}}\left(\frac{5}{6}\right)^{\frac{7}{4}} \sqrt{r+1}\left(1-\frac{1}{7 x^{2}}\right)^{\frac{5}{4}}
$$

Let us now prove some results about these functions. The first one states that they all possess a unique fixed point.

Lemma 3. Let $r \in J$. Then there is a unique $x \in \mathbb{R}_{\geq 1}$ such that $f(x)=r$ and $x$ is a fixed point of $g_{r}$, i.e., $g_{r}(x)=x$.

Proof. As the restriction of $f$ to $\mathbb{R}_{\geq 1}$ is increasing (hence injective) with range $J$, there is a unique $x \in \mathbb{R}_{\geq 1}$ such that $f(x)=r$, or, equivalently, $g_{r}(x)=x$.

In light of Lemma 3 , define a $J$-indexed family $\left(\beta_{r}\right)_{r \in J}$ of real numbers so that $\beta_{r}$ is the unique fixed point of $g_{r}$ for each $r \in J$.

Lemma 4. Let $r \in J$. Then $g_{r}$ is twice differentiable, and for all $x \in \mathbb{R}_{\geq 1}$, 


$$
\begin{aligned}
& g_{r}^{\prime}(x)=\frac{5\left(\frac{7}{6}\right)^{5 / 4}\left(\frac{5}{6}\right)^{7 / 4} \sqrt{r+1}}{14} \cdot \frac{\left(1-\frac{1}{7 x^{2}}\right)^{1 / 4}}{x^{3}}, \\
& g_{r}^{\prime \prime}(x)=\frac{5\left(\frac{7}{6}\right)^{5 / 4}\left(\frac{5}{6}\right)^{7 / 4} \sqrt{r+1}}{28} \cdot \frac{1-6 x^{2}}{x^{6}\left(1-\frac{1}{7 x^{2}}\right)^{3 / 4}} .
\end{aligned}
$$

Hence, $g_{r}^{\prime}$ is positive, so $g_{r}$ is increasing, and $g_{r}^{\prime \prime}$ is negative, so $g_{r}^{\prime}$ is decreasing. Proof. Lemma 4 is an easy exercise in calculus, so we shall omit a proof.

Lemma 5. Let $r \in J$. Then $g_{r}^{\prime}\left(\beta_{r}\right) \leq \frac{5}{12}$.

Proof. Using Lemma 4, observe that

$$
\frac{g_{r}^{\prime}\left(\beta_{r}\right)}{\beta_{r}}=\frac{g_{r}^{\prime}\left(\beta_{r}\right)}{g_{r}\left(\beta_{r}\right)}=\frac{5}{14} \cdot \frac{1}{\beta_{r}^{3}\left(1-\frac{1}{7 \beta_{r}^{2}}\right)},
$$

which yields

$$
g_{r}^{\prime}\left(\beta_{r}\right)=\frac{5}{14} \cdot \frac{\beta_{r}}{\beta_{r}^{3}\left(1-\frac{1}{7 \beta_{r}^{2}}\right)}=\frac{5}{14} \cdot \frac{1}{\beta_{r}^{2}\left(1-\frac{1}{7 \beta_{r}^{2}}\right)}=\frac{5}{14} \cdot \frac{1}{\beta_{r}^{2}-\frac{1}{7}} \leq \frac{5}{14} \cdot \frac{7}{6}=\frac{5}{12},
$$

where we have used the fact that $\beta_{r} \geq 1$.

Lemma 6. Let $r \in J$. Then there is an $\alpha \in\left[1, \beta_{r}\right]$ such that

$$
\mathbb{R}_{\geq \alpha}=\left\{x \in \mathbb{R}_{\geq 1} \mid 0<g_{r}^{\prime}(x) \leq \frac{1}{2}\right\} .
$$

Proof. Lemma 5 says that $g_{r}^{\prime}\left(\beta_{r}\right) \leq \frac{5}{12}<\frac{1}{2}$, so

$$
\beta_{r} \in\left\{x \in \mathbb{R}_{\geq 1} \mid g_{r}^{\prime}(x) \leq \frac{1}{2}\right\} .
$$

The continuity of $g_{r}^{\prime}$ implies that $\left\{x \in \mathbb{R}_{\geq 1} \mid g_{r}^{\prime}(x) \leq \frac{1}{2}\right\}$ is the intersection of $\mathbb{R}_{\geq 1}$ with a closed subset of $\mathbb{R}$, so it is itself a non-empty closed subset of $\mathbb{R}$ bounded below by 1 and therefore has a minimum $\alpha \in\left[1, \beta_{r}\right]$. Then as $g_{r}^{\prime}$ is positive and decreasing, we conclude that

$$
\mathbb{R}_{\geq \alpha}=\left\{x \in \mathbb{R}_{\geq 1} \mid g_{r}^{\prime}(x) \leq \frac{1}{2}\right\}=\left\{x \in \mathbb{R}_{\geq 1} \mid 0<g_{r}^{\prime}(x) \leq \frac{1}{2}\right\} .
$$

The proof of Lemma 6 is complete.

In light of Lemma 6, define a $J$-indexed family $\left(\alpha_{r}\right)_{r \in J}$ of real numbers so that $\mathbb{R}_{\geq \alpha_{r}}=\left\{x \in \mathbb{R}_{\geq 1} \mid 0<g_{r}^{\prime}(x) \leq \frac{1}{2}\right\}$ for each $r \in J$.

Lemma 7. Let $r \in J$. Then $g_{r}$ maps $\mathbb{R}_{\geq \alpha_{r}}$ to itself.

Proof. Observe that $g_{r}(1)=\left(\frac{5}{6}\right)^{7 / 4} \sqrt{r+1} \geq 1$ and

$$
\lim _{x \rightarrow \infty} g_{r}(x)=\left(\frac{7}{6}\right)^{\frac{5}{4}}\left(\frac{5}{6}\right)^{\frac{7}{4}} \sqrt{r+1}
$$


Define a continuous function $h: \mathbb{R}_{\geq 1} \rightarrow \mathbb{R}$ by $h(x):=g_{r}(x)-x$; as $h(1) \geq 0$ and $\beta_{r}$ is the unique root of $h$ (as it is the unique fixed point of $g_{r}$ ), it follows from the inequality $1 \leq \alpha_{r} \leq \beta_{r}$ and the Intermediate Value Theorem that $h\left(\alpha_{r}\right) \geq 0$, i.e., $g_{r}\left(\alpha_{r}\right) \geq \alpha_{r}$. Then as $g_{r}$ is increasing, the image of $\mathbb{R}_{\geq \alpha_{r}}$ under $g_{r}$ is

$$
\left[g_{r}\left(\alpha_{r}\right),\left(\frac{7}{6}\right)^{5 / 4}\left(\frac{5}{6}\right)^{7 / 4} \sqrt{r+1}\left[\subseteq \mathbb{R}_{\geq \alpha_{r}} .\right.\right.
$$

The proof of Lemma 7 is complete.

With Lemma 7, we can finally show that fixed-point iteration is a feasible technique of computing the fixed point of $g_{r}$ for each $r \in J$.

Proposition 3. Let $r \in J$. Then $\sqrt[3]{\left(\frac{7}{6}\right)^{5 / 4}\left(\frac{5}{6}\right)^{7 / 4} \sqrt{r+1}} \in \mathbb{R}_{\geq \alpha_{r}}$, which gives rise to a unique sequence $\left(y_{n}\right)_{n \in \mathbb{N}}$ in $\mathbb{R}_{\geq \alpha_{r}}$ with the following four properties:

- $y_{1}=\sqrt[3]{\left(\frac{7}{6}\right)^{5 / 4}\left(\frac{5}{6}\right)^{7 / 4} \sqrt{r+1}}$

- For all $n \in \mathbb{N}$, we have $y_{n+1}=g_{r}\left(y_{n}\right)$.

- $\lim _{n \rightarrow \infty} y_{n}=\beta_{r}$.

- For all $n \in \mathbb{N}$, we have $\left|y_{n+2}-\beta_{r}\right|<\left(\frac{1}{2}\right)^{n-1}\left(\frac{5}{6}\right)^{7 / 4} \sqrt{r+1}\left(\left(\frac{7}{6}\right)^{5 / 4}-1\right)$.

Proof. Observe that for all $x \in \mathbb{R}_{\geq 1}$,

$g_{r}^{\prime}(x)=\frac{5\left(\frac{7}{6}\right)^{5 / 4}\left(\frac{5}{6}\right)^{7 / 4} \sqrt{r+1}}{14} \cdot \frac{\left(1-\frac{1}{7 x^{2}}\right)^{1 / 4}}{x^{3}} \leq \frac{5\left(\frac{7}{6}\right)^{5 / 4}\left(\frac{5}{6}\right)^{7 / 4} \sqrt{r+1}}{14} \cdot \frac{1}{x^{3}}$

Then as

$$
\sqrt[3]{\left(\frac{7}{6}\right)^{\frac{5}{4}}\left(\frac{5}{6}\right)^{\frac{7}{4}} \sqrt{r+1}} \geq \sqrt[3]{\left(\frac{7}{6}\right)^{\frac{5}{4}}}>1 \text { and } g_{r}^{\prime}\left(\sqrt[3]{\left(\frac{7}{6}\right)^{\frac{5}{4}}\left(\frac{5}{6}\right)^{\frac{7}{4}} \sqrt{r+1}}\right) \leq \frac{5}{14}<\frac{1}{2}
$$

we find that $\sqrt[3]{\left(\frac{7}{6}\right)^{5 / 4}\left(\frac{5}{6}\right)^{7 / 4} \sqrt{r+1}} \in \mathbb{R}_{\geq \alpha_{r}}$, so by the Recursion Theorem, there is a unique sequence $\left(y_{n}\right)_{n \in \mathbb{N}}$ in $\mathbb{R}_{\geq \alpha_{r}}$ such that $y_{1}=\sqrt[3]{\left(\frac{7}{6}\right)^{5 / 4}\left(\frac{5}{6}\right)^{7 / 4} \sqrt{r+1}}$ and $y_{n+1}=g_{r}\left(y_{n}\right)$ for all $n \in \mathbb{N}$.

By definition, $g_{r}$ is bounded in absolute value by $\frac{1}{2}$ on $\mathbb{R}_{\geq \alpha_{r}}$, so the Mean-

Value Theorem implies that for all $p, q \in \mathbb{R}_{\geq \alpha_{r}}$,

$$
\left|g_{r}(p)-g_{r}(q)\right| \leq \frac{1}{2}|p-q|
$$

Hence, by the Banach Fixed-Point Theorem, the restriction of $g_{r}$ to $\mathbb{R}_{\geq \alpha_{r}}$ has a fixed point, which must be $\beta_{r}$; furthermore, $\lim _{n \rightarrow \infty} y_{n}=\beta_{r}$. 
Finally, the Banach Fixed-Point Theorem comes equipped with a suite of inequalities for convergence rates, one of which implies that for all $n \in \mathbb{N}$,

$$
\left|y_{n+2}-\beta_{r}\right| \leq \frac{\left(\frac{1}{2}\right)^{n}}{1-\frac{1}{2}}\left|y_{2}-y_{3}\right|=\left(\frac{1}{2}\right)^{n-1}\left|y_{2}-y_{3}\right| .
$$

As both $y_{2}$ and $y_{3}$ lie in the range of $g_{r}$, and as $g_{r}$ is increasing, it follows that

$$
y_{2}, y_{3} \in\left[g_{r}(1), \lim _{x \rightarrow \infty} g_{r}(x)\left[=\left[\left(\frac{5}{6}\right)^{\frac{7}{4}} \sqrt{r+1},\left(\frac{7}{6}\right)^{\frac{5}{4}}\left(\frac{5}{6}\right)^{\frac{7}{4}} \sqrt{r+1}[.\right.\right.\right.
$$

Therefore,

$$
\left|y_{2}-y_{3}\right|<\left(\frac{7}{6}\right)^{\frac{5}{4}}\left(\frac{5}{6}\right)^{\frac{7}{4}} \sqrt{r+1}-\left(\frac{5}{6}\right)^{\frac{7}{4}} \sqrt{r+1}=\left(\frac{5}{6}\right)^{\frac{7}{4}} \sqrt{r+1}\left(\left(\frac{7}{6}\right)^{\frac{5}{4}}-1\right)
$$

and we conclude that for all $n \in \mathbb{N}$,

$$
\left|y_{n+2}-\beta_{r}\right|<\left(\frac{1}{2}\right)^{n-1}\left(\frac{5}{6}\right)^{\frac{7}{4}} \sqrt{r+1}\left(\left(\frac{7}{6}\right)^{\frac{5}{4}}-1\right)
$$

The proof of Proposition 3 is complete.

In summary, Proposition 3 tells us how to compute $f^{-1}$ on the interval $J$ :

Given $r \in J$, we can recursively construct a convergent sequence $\left(y_{n}\right)_{n \in \mathbb{N}}$ in $\mathbb{R}_{\geq \alpha_{r}}$ such that $y_{1}=\sqrt[3]{\left(\frac{7}{6}\right)^{5 / 4}\left(\frac{5}{6}\right)^{7 / 4} \sqrt{r+1}}$ and $y_{n+1}=g_{r}\left(y_{n}\right)$ for all $n \in \mathbb{N}$. Then the limit of this sequence is $f^{-1}(r)$, with the rate of convergence being at least linear, as guaranteed by the Banach Fixed-Point Theorem.

\section{Static Air Temperature and Total Air Temperature}

The compressibility of air means that air brought to rest by an aircraft's external temperature probe - relative to the aircraft itself — undergoes adiabatic heating. Hence, what the probe measures, called "total air temperature $\left(T_{\mathrm{t}}\right)$ ", is higher than static air temperature $\left(T_{\mathrm{s}}\right)$, which is required to calculate true airspeed. The difference between $T_{\mathrm{s}}$ and $T_{\mathrm{t}}$ can be rather significant at high airspeeds.

$T_{\mathrm{s}}$ depends on $M$ and $T_{\mathrm{t}}$ via the following equation (Trenkle \& Reinhardt, 1973, pp. 31-44):

$$
T_{\mathrm{s}}=\frac{T_{\mathrm{t}}}{1+\frac{\gamma-1}{2} M^{2}}=\frac{T_{\mathrm{t}}}{1+\frac{M^{2}}{5}}
$$

As Mach number depends on static air pressure and impact air pressure, we can define $T_{\mathrm{s}}$ as a function of calibrated airspeed, pressure altitude, and $T_{\mathrm{t}}$.

Definition 7. Static air temperature is the function

$$
T_{\mathrm{s}}: \mathbb{R}_{\geq 0} \times\left[0 \mathrm{~m}, 20000 \mathrm{~m}\left[\times \mathbb{R}_{\geq 0} \rightarrow \mathbb{R}_{\geq 0}\right.\right.
$$


defined by

$$
T_{\mathrm{S}}\left(\mathrm{CAS}, h, T_{\mathrm{t}}\right):=\frac{T_{\mathrm{t}}}{1+\frac{M(\mathrm{CAS}, h)^{2}}{5}} .
$$

Note that $T_{\mathrm{s}}$ is continuous.

\section{Computing True Airspeed}

True airspeed can be computed from Mach number once the local speed of sound $a$ is known. It is known that $a$ varies directly with the square root of $T_{\mathrm{s}}$, so TAS depends on $M$ and $T_{\mathrm{s}}$ via the following equation (Herrington et al., 1966, pp. 12-23):

$$
\text { TAS }=a M=a_{0} M \sqrt{\frac{T_{\mathrm{S}}}{T_{0}}} .
$$

Combining all the earlier results of this section, this equation allows us to define true airspeed as a function of calibrated airspeed, pressure altitude, and $T_{\mathrm{t}}$. Definition 8. True airspeed is the function

defined by

$$
\text { TAS: } \mathbb{R}_{\geq 0} \times\left[0 \mathrm{~m}, 20000 \mathrm{~m}\left[\times \mathbb{R}_{\geq 0} \rightarrow \mathbb{R}_{\geq 0}\right.\right.
$$

$$
\operatorname{TAS}\left(\mathrm{CAS}, h, T_{\mathrm{t}}\right):=a_{0} M(\mathrm{CAS}, h) \sqrt{\frac{T_{\mathrm{s}}\left(\mathrm{CAS}, h, T_{\mathrm{t}}\right)}{T_{0}}} .
$$

Note that TAS is continuous.

\section{Conclusion}

Through various mathematical techniques, the question of the existence of solutions of a mathematical WTP3 (TAS,WS, $\theta, \varphi$ ) can be reduced to a question about the ordered pair $\left(\cos (\varphi-\theta), \frac{\mathrm{WS}}{\mathrm{TAS}}\right)$, which is whether it is an element of the set $S$ (the go condition) or not (the no-go condition). Examples 1 and 2 show how easy it is to test the go/no-go conditions graphically. Hopefully, our results offer pilots a practical method of evaluating the feasibility of their flight plan, based on wind conditions.

As the aviation industry readies itself for a wave of projects on supersonic passenger flight in the coming years (O'Hare, 2021), it is naturally expected that high-speed flight will no longer be strictly within the purview of military aviators. With the goal of equipping pilots from all backgrounds with practical knowledge about high-speed flight, we constructed a function that calculates true airspeed in terms of only calibrated airspeed, pressure altitude, and total air temperature. This function has great theoretical importance because it allows a pilot to calculate true airspeed using data available from only a standard instrument panel without direct knowledge of impact and static air pressures, even in supersonic flight. However, this function is not known to have a fully explicit description in terms of the usual arithmetic operations and elementary functions, so a numerical technique must be 
applied to evaluate it for a certain set of inputs. Not only were we able to establish that fixed-point iteration works, but we were also able to offer error estimates. We believe that such a complete and rigorous analysis is the first of its kind.

The tools introduced in this paper will help pilots buttress their knowledge of aerial navigation, aircraft instrumentation, and aeronautics. 


\section{References}

Air Training Command. (1973). Air Navigation. Flying Training. US Government Printing Office.

Brown, F. S. (2012). Subsonic Relationships Between Pressure Altitude, Calibrated Airspeed, and Mach Number. (No. AFFTC-TIH-10-01). United States Air Force. https://doi.org/10.21236/ada569074

Daidzic, N. E. (2015). General solution of the wind triangle problem and the critical tailwind angle. International Journal of Aviation Sciences, 1(1), 57-93.

Federal Aviation Administration. (2016). Pilot's Handbook of Aeronautical Knowledge. FAA. U.S. Department of Transportation, Federal Aviation Administration, Flight Standards Service.

Herrington, R. M., Schoemacher, P. E., Bartlett, E. P., \& Dunlap, E. W. (1966). Flight Test Engineering Handbook. Edwards AFB, CA: Air Force Flight Test Center. https://doi.org/10.21236/ad0636392

Miller, S. A., \& Veltin, J. (2012). Experimental and Numerical Investigation of Flow Properties of Supersonic Helium-Air Jets. AIAA Journal, 49(1), 235246. https://doi.org/10.2514/1.j050720

NASA. (1966). X-15 \#3 Control Panel. [Photograph]. Retrieved from https://www.nasa.gov/ centers/dryden/multimedia/imagegallery/X15/EC66-1206.html

O'Hare, M. (2021, May 18). Boom Supersonic aims to fly 'anywhere in the world in four hours for $\$ 100^{\prime}$. CNN Travel. Retrieved from http://edition.cnn.com/travel/article/boom-supersonic-four-hours-100bucks/index.html

Porter, F. H., \& Hartman, R. D. (1979). (rep.). Calibrated Airspeed and the "F" Factor. (No. USAFA-TR-80-7). United States Air Force Academy.

Trenkle, F., \& Reinhardt, M. (1973). In-Flight Temperature Measurements. North Atlantic Treaty Organization, 2(160).

US Government Printing Office. (1976). US Standard Atmosphere, 1976. NASA Technical Reports Server.

US Navy. (2017). Introduction to Air Navigation. United States Department of Defense.

Wiolland, K. (2005, January). Air Data Computers. The Aircraft Electronics Association. 